\title{
Utilizing Citizen Science to Reverse the Current Lead Testing Paradigm: Development of a Scalable, Low-cost Home Lead Test Kit
}

Katlyn Sawyer ${ }^{1}$, Chris Knaub², Meghanne Tighe ${ }^{3}$, Danielle Forbes ${ }^{5}$, Claire Marks ${ }^{6}$, Lane Nicolay ${ }^{6}$, Citlali Gutierrez ${ }^{7}$, Mike Dowd $^{4}$, Maggie Bielski ${ }^{6}$, Matthew Sisk ${ }^{8}$, Michelle Ngai $^{2}$, Marya Lieberman ${ }^{3}$, Graham Peaslee ${ }^{9}$, Heidi Beidinger ${ }^{2}$

1 Indiana University School of Medicine, 2 University of Notre Dame, Eck Institute for Global Health, 3 University of Notre Dame, Department of Chemistry, 4 University of Hawaii, Manoa, 5 University of Toledo, 6 University of Notre Dame, 7 Chipola College, 8 University of Notre Dame, Center for Digital Scholarship, 9 University of Notre Dame, Department of Physics

Background: The Center for Disease Control recommends case management begin at a blood lead level of $5 \mu \mathrm{g} / \mathrm{dL}$, yet Indiana does not take action until a blood lead level of $10 \mu \mathrm{g} / \mathrm{dL}$. Low levels of lead can cause irreversible neurological damage in children. The goal of this study was to design a scalable, low-cost Home Lead Test Kit to proactively find lead in homes.

Methods: Individuals were recruited through community partnerships, community lead testing events, and flyers. Qualitative data was recorded during home visits as participants used the kit. An x-ray fluorescence analyzer was used in the field and laboratory to analyze lead levels of samples. Results were blinded during the analysis.

Results: To date, 40 homes have been recruited, and 20 have been completed. The average completion time of the kit was 23.45 minutes. Of the 8 pre-1950 homes $100 \%$ had elevated lead results, of the $71950-1978$ homes $43 \%$ had elevated lead results, and of the 5 post 1978 homes $0 \%$ had elevated lead results.

Conclusion and Potential Impact: The study is ongoing. Preliminary results support the hypothesis that pre-1950 homes have a high risk of lead exposure, and post 1978 homes have minimal to no levels of lead. Feedback from study participants regarding kit usability has been positive. Future plans are to produce a kit to be scaled up in St. Joseph County with the goal of a statewide model. This kit may allow citizens to identify lead hazards to prevent children from lead exposure. 